\title{
Challenging the role of implicit processes in probabilistic category learning
}

\author{
BEN R. NEWELL \\ University of New South Wales, Sydney, New South Wales, Australia \\ AND \\ DaVId A. Lagnado and DaVid R. Shanks \\ University College London, London, England
}

\begin{abstract}
Considerable interest in the hypothesis that different cognitive tasks recruit qualitatively distinct processing systems has led to the proposal of separate explicit (declarative) and implicit (procedural) systems. A popular probabilistic category learning task known as the weather prediction task is said to be ideally suited to examine this distinction because its two versions, "observation" and "feedback," are claimed to recruit the declarative and procedural systems, respectively. In two experiments, we found results that were inconsistent with this interpretation. In Experiment 1, a concurrent memory task had a detrimental effect on the implicit (feedback) version of the task. In Experiment 2, participants displayed comparable and accurate insight into the task and their judgment processes in the feedback and observation versions. These findings have important implications for the study of probabilistic category learning in both normal and patient populations.
\end{abstract}

In recent years, there has been considerable interest in the hypothesis that different cognitive tasks recruit qualitatively distinct processing systems (Ashby, Alfonso-Reese, Turken, \& Waldron, 1998; Knowlton, Squire, \& Gluck, 1994; Squire, 2004). The major distinction drawn by proponents of this view is between an explicit or declarative system that requires awareness and involves analytic processing and an implicit or procedural system that operates in the absence of conscious awareness.

This dichotomous view of cognition naturally invites experimental manipulations hypothesized to have differential effects on the two putative systems (e.g., Ashby \& Maddox, 2005). We drew on this strategy and scrutinized the processes underlying performance in a task that has been one of the primary sources of data for the proposed dissociation between the two systems. The task is a probabilistic category learning task known as the weather prediction task (WPT); (Knowlton et al., 1994). The task has been used in numerous investigations with a variety of patient populations and unimpaired individuals, and has therefore been highly influential in the development of theoretical models of dissociable learning and memory systems (Squire, 2004).

In the WPT people learn gradually, over a series of trials, to predict a binary outcome on the basis of four binary cues. Learning the probabilistic cue-outcome relations is claimed to be mediated primarily via the procedural system (Gluck, Shohamy, \& Myers, 2002; Knowlton et al., 1994; Poldrack et al., 2001; Shohamy, Myers, Onlaor, \& Gluck, 2004).
In Experiment 1, we tested the prediction that performance in this task, if mediated by a procedural system, should be relatively unaffected by placing additional demands on working memory. This is because execution of a working memory task should be performed primarily by the declarative system, allowing learning of the cueoutcome relations in the WPT to proceed normally (Foerde, Poldrack, \& Knowlton, 2007).

We used a concurrent task that has been used by multiple-systems theorists to provide evidence for the operation of explicit processes in a primary task. Specifically, Waldron and Ashby (2001) and Zeithamova and Maddox (2006) demonstrated that primary task performance of participants who were given the concurrent numerical Stroop task that we used was more affected if the primary task (categorization) was performed optimally by a declarative system than if it was performed optimally by a procedural system. Accordingly, in our experiment any interference effects of the concurrent task on WPT performance can be taken as evidence of the involvement of a declarative system; see Nosofsky and Kruschke (2002) for an alternative interpretation of Waldron and Ashby.

In Experiment 2, we compared the standard "feedback" version of the WPT with an "observation" version, which presented participants, on each trial, with both the cues and the outcome simultaneously. This paired-associate arrangement does not rely on trial-by-trial feedback; therefore, learning the task is claimed not to recruit the procedural system, because there is no "surprising" reward

B. R. Newell, ben.newell@unsw.edu.au 
associated with learning (Ashby et al., 1998). Rather, the declarative system is thought to be primarily responsible for learning (Poldrack et al., 2001). Given this interpretation, a straightforward prediction is that participants trained under observation conditions should exhibit better explicit knowledge than those who learn the procedurally mediated feedback version.

Numerous conclusions about the operation of distinct systems in probabilistic category learning rest on results from experiments involving the WPT. For example, Shohamy et al. (2004) demonstrated that Parkinson's patients were impaired in learning the feedback version, but that they were unimpaired, relative to controls, on the observation version. Similarly, Poldrack et al. (2001) demonstrated equivalent performance in normals on feedback and observation versions of the task, but presented neuroimaging data that suggested differential engagement of the basal ganglia and of the medial temporal lobe in the different versions.

In experiments of this type, different patterns of performance are taken (time and again) to provide evidence for the existence of dissociable learning (and memory) systems. Very rarely is the notion entertained that a single, declarative system might be able to provide an equally good and potentially more parsimonious account of the data (Lagnado, Newell, Kahan, \& Shanks, 2006; Zaki, 2004).

We aimed to redress this balance by testing the two predictions described above that arise from the multiplesystems view. Finding support for these two predictions would help to justify the extensive use of the WPT in cognitive neuroscience as a "tool" for demonstrating the operation of the hypothesized procedural and declarative systems. Finding results inconsistent with the predictions would suggest a reevaluation of the claims made for the characteristics of the two systems, which have, in considerable part, been based on particular interpretations of performance in the WPT.

\section{EXPERIMENT 1}

\section{Method \\ Participants \\ Twenty-four undergraduate students from the University of New} South Wales (average age, 20.8; age range, 17-42; $S D=5.5$ ) participated in the experiment in return for course credit.

\section{Design and Materials}

There were two groups, one trained only on the WPT, and the other given the WPT and a concurrent memory load task. In the WPT, the stimuli were drawn from a set of four cards, each with a different geometric pattern (squares, diamonds, circles, triangles). During training, participants saw a total of 102 trials, on each of which they were presented with a pattern of one, two, or three cards. Each trial was associated with one of two outcomes (Rain or Fine); overall, these two outcomes occurred equally often. The 14 pattern frequencies are shown in Table 1, along with the frequency of each outcome for each pattern. The learning set was constructed so that each card was associated with the outcome with a different independent probability (see Table 1).

Participants experienced identical pattern frequencies (order randomized for each participant), but because the actual outcome for each pattern was determined probabilistically, experienced outcomes could differ slightly across participants.

\section{Procedure}

Training phase. In the training phase, participants were told that on each trial their task was to decide whether the combination of cards presented predicted rainy or fine weather. After making their prediction, participants received immediate feedback as to the actual weather on that trial, and whether they were correct or incorrect.

Memory load task. The concurrent task required participants to remember which of two numbers was numerically larger and which was physically larger. On $50 \%$ of trials, both the size and value dimensions were congruent; on $50 \%$, they were incongruent. The numbers appeared simultaneously on either side of the card combinations and remained on screen for $1 \mathrm{sec}$. Physically large numbers appeared in 24-point font, small in 14-point font.

The ordering of events on each trial was as follows: (1) cards and numbers presented; (2) weather prediction made; (3) feedback on prediction received; (4) questions about size or value of numbers presented and answered; (5) feedback on number response received.

Test phase. All participants were asked to give probability ratings for each of the four cards in response to the question "On the basis of this card what do you think the weather is going to be like?" They registered their rating using a continuous slider scale ranging from "Definitely fine" to "Definitely rainy," with "As likely fine as rainy" as the midpoint. Finally, all participants performed 42 trials $(14$ patterns $\times 3)$ of the WPT without the concurrent memory load task and without corrective feedback.

\section{Results and Discussion}

Accuracy in the concurrent memory task was high, with an average across the 102 trials of $85.3 \%(S D=11.3)$. Participants in both groups improved in their ability to predict the outcome across training trials. The concurrent group improved from a mean of $57.3 \%$ correct predictions across the first 25 trials to a mean of $63.2 \%$ across the final 25 . (Correct predictions in training and test refer to the proportion of trials on which the prediction matched the probabilistically determined outcome.) The control group improved from

Table 1

Pattern and Outcome Frequencies Used in Experiments 1 and 2

\begin{tabular}{crrrrrrrr}
\hline & \multicolumn{1}{c}{ Cards Present } & & & \\
\cline { 2 - 5 } Pattern & $\mathrm{C}_{1}$ & $\mathrm{C}_{2}$ & $\mathrm{C}_{3}$ & $\mathrm{C}_{4}$ & & Rain & Fine & Total \\
\hline A & 0 & 0 & 0 & 1 & 9 & 1 & 10 \\
B & 0 & 0 & 1 & 0 & 4 & 1 & 5 \\
C & 0 & 0 & 1 & 1 & 12 & 1 & 13 \\
D & 0 & 1 & 0 & 0 & 1 & 4 & 5 \\
E & 0 & 1 & 0 & 1 & 5 & 1 & 6 \\
F & 0 & 1 & 1 & 0 & 2 & 2 & 4 \\
G & 0 & 1 & 1 & 1 & 8 & 1 & 9 \\
H & 1 & 0 & 0 & 0 & 1 & 9 & 10 \\
I & 1 & 0 & 0 & 1 & 2 & 2 & 4 \\
J & 1 & 0 & 1 & 0 & 1 & 5 & 6 \\
K & 1 & 0 & 1 & 1 & 2 & 2 & 4 \\
L & 1 & 1 & 0 & 0 & & 1 & 12 & 13 \\
M & 1 & 1 & 0 & 1 & 2 & 2 & 4 \\
N & 1 & 1 & 1 & 0 & 1 & 8 & 9 \\
Total & & & & & 51 & 51 & 102 \\
\hline
\end{tabular}

Note-1, card present; 0 , card absent; $\mathrm{C}_{1}-\mathrm{C}_{4}$, Cards $1-4$, respectively. Cue validities are calculated from pattern frequencies and conditional probabilities. For example, the seven patterns $(\mathrm{H}$ to $\mathrm{N})$ in which $\mathrm{C}_{1}$ is present appeared a total of 50 times in the experiment, and on 10 of these occasions (on average) the outcome was Rain; thus, the probability of Rain given the presence of $C_{1}$ was $10 / 50(.20)$. The assignment of shape type (circles, squares, etc.) to $\mathrm{C}_{1-4}$ was constant across participants, but the positions of cards on the screen were counterbalanced. 
Table 2

\begin{tabular}{|c|c|c|c|c|c|c|c|c|}
\hline & \multicolumn{2}{|c|}{ Card 1} & \multicolumn{2}{|c|}{ Card 2} & \multicolumn{2}{|c|}{ Card 3} & \multicolumn{2}{|c|}{ Card 4} \\
\hline & $M$ & $S D$ & $M$ & $S D$ & $M$ & $S D$ & $M$ & $S D$ \\
\hline Objective probability of rain given single card & .20 & & .40 & & .60 & & .80 & \\
\hline \multicolumn{9}{|c|}{ Experiment 1} \\
\hline Subjective rating, control group & .09 & .24 & .43 & .34 & .50 & .29 & .82 & .28 \\
\hline Subjective rating, concurrent group & .28 & .24 & .43 & .34 & .48 & .35 & .75 & .28 \\
\hline \multicolumn{9}{|c|}{ Experiment 2} \\
\hline Subjective rating, feedback group & .16 & .15 & .43 & .27 & .65 & .21 & .87 & .19 \\
\hline Subjective rating, observation group & .07 & .14 & .42 & .26 & .53 & .55 & .94 & .06 \\
\hline
\end{tabular}

$64.1 \%$ to $70.8 \%$ over the same trials. The improvements were of approximately the same magnitude, but a linear trend test showed that the improvement was only significant for the control group $[F(1,23)=4.37, p<.05]$, not for the concurrent group $[F(1,23)=2.17, p>.09]$. Moreover, a comparison of performance collapsed across the second half of the training trials showed a significantly higher number of correct predictions in the control group $(M=71.6 \%)$ than in the concurrent group $(M=62.4 \%)[F(1,23)=4.34$, $p<.05]$. This pattern of results indicates that the concurrent task significantly affected participants' ability to learn the WPT via feedback. The concurrent group did still learn; across training they performed at significantly above the chance level of $50 \%[t(11)=2.83, p<.05]$.

Table 2 displays the mean probability ratings of rain for each card. A 2 (group) $\times 4$ (card type) ANOVA on the ratings indicated a significant effect of card type $[F(3,66)=$ $16.56, p<.001]$, demonstrating clear discrimination between the objective probabilities associated with each card. There was no effect of group and no interaction $\left(F_{\mathrm{S}}<1\right)$. Contrasts comparing ratings for each card revealed a significant difference only for Card $1[t(22)=2.35, p=$ $.028 ; p$ values for other three contrasts, all $>.53]$. Control participants underestimated the objective probability of Card 1, whereas concurrent participants overestimated it.

In the test phase, the concurrent group achieved 58.3\% correct predictions $(S D=11.6)$, whereas the control group achieved 73.0\% $(S D=8.1)[F(1,22)=12.88, p<$ $.05]$. The persistence of the deficit in performance across the single-task test trials suggests that participants given the concurrent task during training were not simply impaired in the expression of knowledge at training but in the acquisition of that knowledge (Foerde et al., 2007). Performance in both conditions was significantly above chance level $(50 \%)$ [concurrent, $t(11)=2.49, p<.05$ ].

\section{Strategy Analyses}

Gluck et al. (2002) identified three classes of strategies, differing primarily in their complexity, which participants might use to "solve" the weather prediction task.

Multicue maximizing strategy. This is the optimal strategy for learning the task. It involves responding to each pattern with the outcome most often associated with that pattern.
One-cue strategy. This is a suboptimal strategy in which participants, disregarding other cues, respond on the basis of the presence or absence of a single cue.

Singleton strategy. This is another suboptimal strategy, in which a participant learns only the responses associated with the patterns on which a single card appears (Patterns A, B, D, and $\mathrm{H}$ in Table 1) and guesses on the remaining trials.

We also considered a multicue matching strategy (Lagnadoet al., 2006), which assumes that participants distribute their responses to a pattern according to the actual probabilities associated with that pattern. For example, for Pattern A across the 102 training trials, a participant would respond "rain" 9 times and "fine" once, in contrast to the 10:0 "rain" responses predicted by the multicue maximizing strategy.

We used the same method as Gluck et al. (2002) to fit participants' learning profiles to the strategies described above. The basic procedure was to calculate the degree to which each model fit the participant's data using a least mean squares (LMS) measure, with 0.00 indicating a perfect fit (Gluck et al., 2002). Lagnado et al. (2006) discussed the limitations of Gluck's strategy fitting methods - for example, the assumption of perfect cue validity knowledge on Trial 1 of the experiment - and developed a more fine-grained dynamic method for assessing "strategy" use. This new procedure was not appropriate for the present experiments, because, in part, of the shorter training sequences employed here; so, despite its limitations, we relied on the Gluck et al. methodology.

Figure 1 (left panel) shows the distribution of strategies across participants in the two groups for the 102 training trials. Participants were classified simply by allocating them to the best fitting (i.e., lowest LMS) strategy; see Figure 1, which highlights the dominance of the multimatch strategy in the control group and the adoption of simpler strategies in the concurrent group. The distribution of strategies differed significantly between the two groups $\left[\chi^{2}(3)=6.7, p<.05\right]$.

The distribution of strategies is inconsistent with the idea that learning the WPT through feedback is unaffected by the addition of a concurrent task. On the contrary, there appears to be a qualitative shift in the kinds of strategies that participants use in the two conditions. 

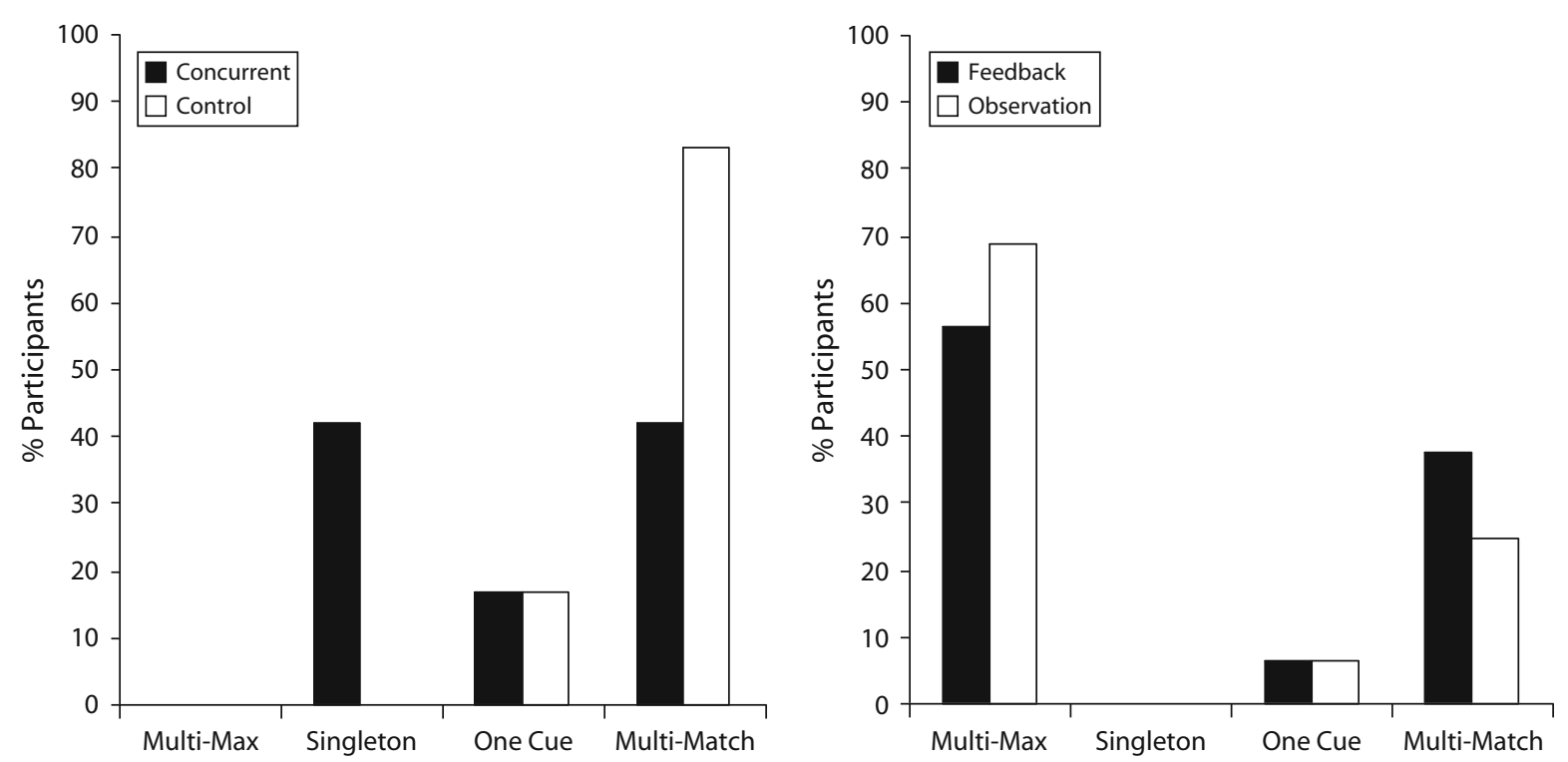

Figure 1. Left panel, Experiment 1: Strategy allocation during training trials for participants trained in the feedback task under either concurrent task or control (no concurrent task) conditions. Right panel, Experiment 2: Strategy allocation during test trials following either observation or feedback training. Note that Gluck et al. (2002) suggested a tolerance level of 0.10 as indicative of a "fit" with one of the models (i.e., consistent with a model); other studies have simply used the lowest score to indicate a "best fit" (Hopkins, Myers, Shohamy, Grossman, \& Gluck, 2004). In Experiment 1, the "fits" lay between 0.00 and 0.16, with three participants (from the concurrent group) rating above 0.10 ; these three were allocated to the strategy with the lowest LMS. In Experiment 2, fits ranged between 0.00 and 0.20 . Six participants rated above 0.10 and were allocated to the strategy with the lowest LMS.

\section{EXPERIMENT 2}

The claim that the observation version of the WPT relies primarily on a declarative system leads to the prediction that participants trained under observation conditions should exhibit better explicit knowledge than should participants who learn the procedurally mediated feedback version (Gluck et al., 2002; Shohamy et al., 2004). Learning in the procedural system is hypothesized to be unconscious. Some researchers also suggest that qualitatively different strategies are used to solve the two versions of the task (Poldrack et al., 2001). Experiment 2 tested these predictions by comparing a group trained under feedback conditions with a group trained under observation conditions.

\section{Method}

Participants. Thirty-two undergraduates from the University of New South Wales (average age, 20.1; age range, $18-27 ; S D=2.1$ ) participated in the experiment in return for course credit.

Design and Procedure. There were two groups. One group learned the observation version of the WPT, and the other group learned the feedback version. In the observation version, both the cards and the outcome were presented simultaneously on every trial. Participants were told to observe and to try to learn which combinations of cards were associated with rainy or fine weather.

The different procedures in the observation and feedback tasks necessitated different questions for assessing participants' insight. In the feedback group, participants made trial-by-trial predictions, enabling questions to be asked about insight into their own judgment process. In contrast, in the observation group, participants did not make predictions because cues and outcomes were presented simultaneously, so questions were asked about insight into the task structure (Lagnado et al., 2006).
In the feedback group, participants made a prediction and were then asked, "How much did you rely on each card in making your prediction?" The response options were "Greatly," "Moderately," "Slightly," or "Not at all." Participants received corrective feedback on the outcome. On each trial, participants in the observation group were asked, "How important is each card in determining the outcome?" and they used the same 4-point rating scale to make a response. If performance is mediated by an implicit mechanism, there should be no divergence between the ratings made for strong and weak predictors; in contrast, an explicit mechanism predicts clear divergence. Note that using online ratings of insight does not appear to affect the level of accuracy achieved, nor does it appear to affect the strategies adopted in solving the feedback version of the task (Lagnado et al., 2006).

As opposed to the single retrospective assessment made in Experiment 1 , probability ratings for each of the four cards were made after 51 and 102 trials. Following the second set of ratings, all participants completed 42 test trials, in which they made predictions but were not provided with feedback.

\section{Results and Discussion}

The mean proportion of correct predictions in the feedback group was $66 \%$ across the first 25 trials and $74.3 \%$ across the final 25 , linear trend $[F(1,15)=9.87, p<$ $.05]$.

At test, the mean proportion of correct predictions was $73.3 \%(S D=7.0)$ after feedback training and $73.2 \%$ $(S D=6.9)$ following observation training. This pattern of identical performance is consistent with previous studies comparing observation and feedback versions of the WPT (Poldrack et al., 2001; Shohamy et al., 2004).

The mean probability ratings of rain for each card (collapsed across those taken at Trials 51 and 102) are shown 
in Table 2. A 2 (group) $\times 4$ (card type) ANOVA on these ratings revealed a main effect of card type $[F(3,90)=$ $80.6, p<.001]$. Importantly, the effect of group and the interaction were not significant (both $p \mathrm{~s}>.20$ ). Furthermore, contrasts comparing ratings for each card revealed no significant group differences (all $p s>.10$ ). Because these analyses do not incorporate the ordering of the independent variables (i.e., cards), we also computed a regression slope for each participant. ${ }^{1}$ The mean slope values were $0.27(S D=.09)$ and $0.24(S D=.09)$ for the observation and feedback groups, respectively. These means were not statistically different $[t(30)=1.07$, $p>.20]$, suggesting that both groups were equally able to discriminate among the cards. Finally, we examined the mean absolute difference between the rating provided for each card (collapsed across the Trials 51 and 102 ratings) and the objective probability for each card. Difference scores were calculated for each card for each participant and then averaged to produce a mean overall absolute difference between objective and subjective ratings. The difference score was .17 for the observation group and .18 for the feedback group; in other words, there was essentially no difference in calibration between the two groups. $^{2}$

For the cue reliance (feedback group) and cue importance (observation group) ratings, we analyzed only those trials on which more than one card was present and collapsed across the ratings given for "strong" (Cards 1 and 4) and "weak" (Cards 2 and 3 ) predictors. Figure 2 plots the mean reliance ratings (feedback group) and mean impor- tance ratings (observation group) for strong and weak cards collapsed across 20 blocks of training trials $(19 \times 5$ trials, $1 \times 7$ trials). The ratings provided by each group were analyzed separately because of the difference in the nature of the question asked on each trial (i.e., reliance on a card vs. importance for determining the outcome).

Feedback. A 2 (card type: strong vs. weak) $\times$ 20 (block) ANOVA on the cue reliance ratings revealed a main effect of card type $[F(1,15)=17.6, p<.002]$ indicating discrimination between reliance on strong and weak cues; a main effect of block $[F(19,285)=2.10, p<$ $.005]$ and an interaction $[F(19,285)=2.55, p<.001]$. Using a criterion that required the mean ratings for strong and weak cards to be significantly different (at $\alpha=.05$ ), for two consecutive blocks, significant divergence was found after 9 blocks (45 trials).

Observation. Analysis of the cue importance ratings revealed a main effect of card type $[F(1,15)=55.1, p<$ $.001]$, no effect of block $[F(19,285)=.742, p>.50]$, and an interaction $[F(19,285)=1.97, p<.01]$. Significant divergence between strong and weak cards was found after 6 blocks (30 trials).

An important question here is whether one should interpret the slightly earlier divergence of the observation group as indicative of the operation of a different system in this group. A dual-system theorist may indeed take such a line, but we argue that it constitutes very weak evidence for such a claim, when taken in conjunction with the identical levels of performance, lack of difference in the blocked probability ratings, and similar

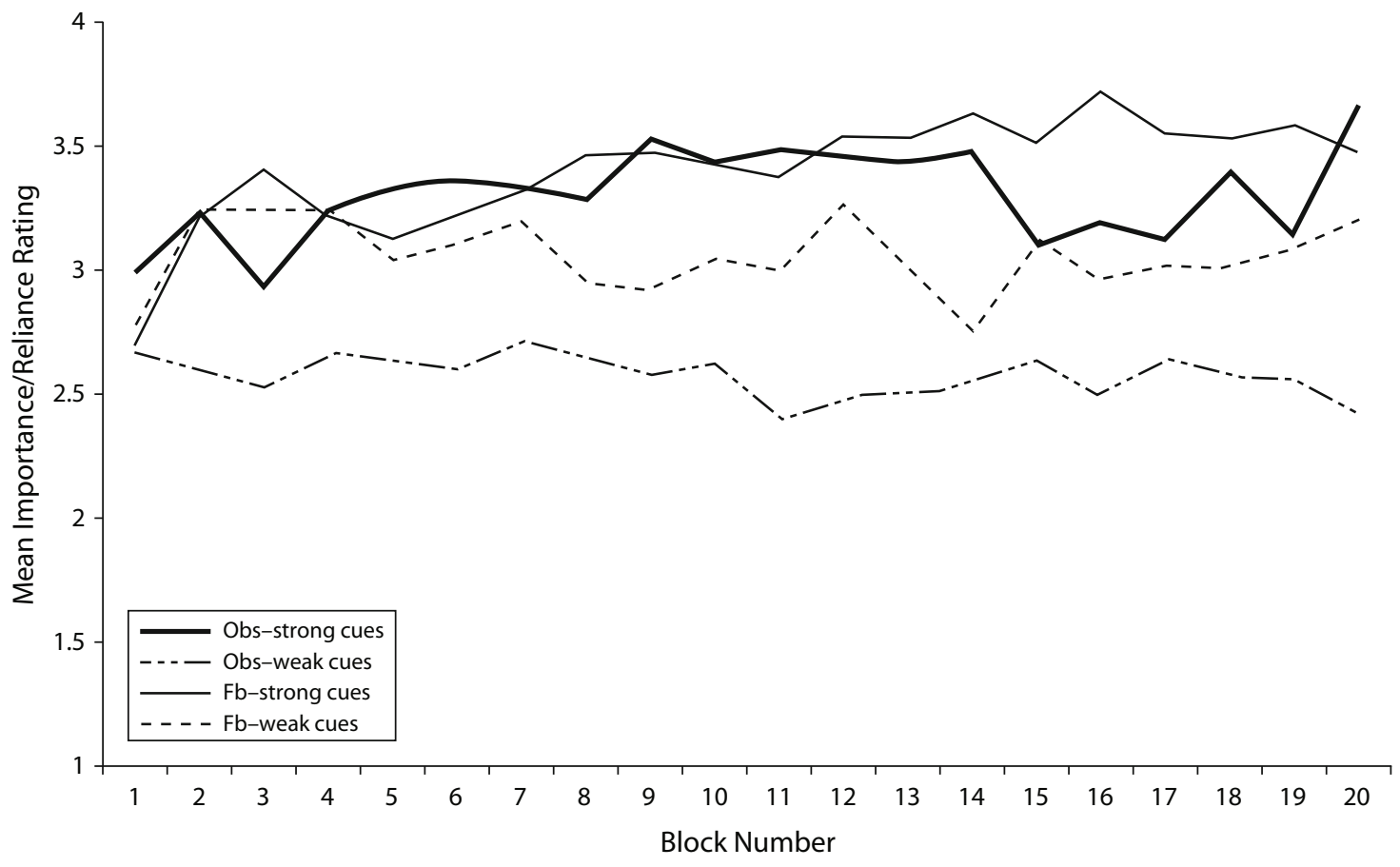

Figure 2. Experiment 2: Mean trial-by-trial cue importance (observation group) and cue reliance (feedback group) ratings averaged across blocks of trials. The heavy lines are ratings made by observation participants, the softer ones those made by feedback participants. The dashed lines indicate ratings collapsed across the two weaker predictors, the solid lines ratings collapsed across the two stronger predictors. 
strategy allocations in the two versions of the task (see below). The key result here is that in both groups there is clear evidence for a divergence between the ratings provided for strong and weak cards, indicating that both tasks rely on explicit knowledge. The earlier divergence seen in the observation group suggests a slightly different time course for the development of insight into the task structure (i.e., the importance of cards for determining outcomes) and the judgment process (i.e., the reliance on cards for making predictions) (Lagnado et al., 2006) but does not, to our minds, imply the operation of a different system. Note that these results extend the recent findings of Lagnado et al. by comparing insight in observation and feedback participants.

\section{Strategy Analyses}

Figure 1 (right panel) shows the distribution of strategies for the 42 test trials following observation and feedback training (analyses could not be done for the training trials because no predictions are made under observation training). The more complex strategies again clearly dominate in both groups. There is little suggestion that qualitatively different strategies are employed in the feedback and observation conditions $\left[\chi^{2}(3)=.60, p>.5\right]$.

\section{GENERAL DISCUSSION}

In two experiments, we obtained results at odds with those predicted by a dual-systems view of probabilistic category learning. In Experiment 1, the introduction of a concurrent memory load had a significant impact on learning in a prototypical procedurally mediated task, and in Experiment 2 we found similar and accurate levels of insight in both the putatively procedural and declarative versions of the task.

Strategy analyses revealed in Experiment 1 that the addition of a concurrent memory load reduced the number of participants relying on complex multicue strategies. This result was predicted by the operation of a working memory dependent declarative system but not by a working memory independent procedural system. Strategy analyses of the data from Experiment 2 provided no evidence to suggest that qualitatively different strategies were used to solve the two versions of the task.

These results challenge the interpretation of the WPT as a procedurally mediated task and, more importantly, undermine the claims made by multiple-systems theorists based on previous interpretations of the task. For example, the claim made on the basis of imaging data by Poldrack et al. (2001) - that learning in the feedback and observation versions recruits qualitatively different systems - may need to be reevaluated, given our finding of identical performance, comparable levels of insight, and similar strategy distributions in the two versions. Although imaging data may provide valuable evidence concerning the brain regions associated with different tasks, differences in activation do not by themselves necessitate the operation of distinct systems (Sherry \& Schacter, 1987).
We suggest that the most parsimonious explanation of our results is that performance in both versions of the task is mediated by a single explicit learning mechanism: A single declarative learning process drives participants' behavioral responses (i.e., their online predictions), their explicit judgments about the task structure (i.e., their blocked probability ratings), and their explicit judgments about their own cue usage or cue importance (i.e., their online cue ratings).

A single system account predicts that when participants' attention is drawn to a concurrent task (as in Experiment 1), fewer resources will be available for learning the cueoutcome contingencies in the WPT. As a consequence, predictions should be less accurate and participants should be less able to adopt complex strategies. Both of these predictions are clearly supported by the results of Experiment 1. A single system account also predicts the pattern of data found in Experiment 2. If, as we suggest, both the observation and feedback versions are mediated by the same explicit mechanism, one would expect the similar levels of performance, insight into task structure and cue reliance, and comparable adoption of strategies that we observed across the two versions of the task.

A single-system account also needs to address the evidence for dissociations that come from the neuropsychological studies that have used the WPT (Shohamy et al., 2004). It is beyond the scope of this article to discuss all the relevant data, but we would argue that in many cases (especially as far as Parkinson's patients and amnesiacs are concerned), dissociations in performance may be more parsimoniously accounted for by a generalized learning decrement than by disruptions to qualitatively distinct systems (Lagnado et al., 2006; Zaki, 2004).

We scrutinized a paradigm procedural task that has been used extensively in cognitive neuroscience to support the claim for dissociable learning systems. In two experiments, we found little evidence of the expected "signatures" of implicit performance, and we suggest that performance in both the observation and feedback versions of the WPT is most parsimoniously explained by the operation of a single, explicit learning mechanism. Given these and related recent results (Lagnado et al., 2006) we suggest a reevaluation of the multiple-systems theorizing made on the basis of previous interpretations of the task.

\section{AUTHOR NOTE}

The support of the Australian Research Council (Grant DP055818) is gratefully acknowledged. We thank Elia Vecellio and Tamara Cavenett for assistance in data collection and Russ Poldrack, Safa Zaki, and Martin Meeter for comments on an earlier version of the article. All correspondence concerning this article should be addressed to B. R. Newell, School of Psychology, University of New South Wales, Sydney 2052, New South Wales, Australia (e-mail: ben.newell@unsw.edu.au).

\section{REFERENCES}

Ashby, F. G., Alfonso-Reese, L. A., Turken, A. U., \& Waldron, E. M. (1998). A neuropsychological theory of multiple systems in category learning. Psychological Review, 105, 442-481.

Ashby, F. G., \& Maddox, W. T. (2005). Human category learning. Annual Review of Psychology, 56, 149-178.

Foerde, K., Poldrack, R. A., \& Knowlton, B. J. (in press). Secondary task effects on classification learning. Memory \& Cognition. 
Gluck, M., Shohamy, D., \& Myers, C. (2002). How do people solve the "weather prediction" task? Individual variability in strategies for probabilistic category learning. Learning \& Memory, 9, 408-418.

Hopkins, R. O., Myers, C. E., Shohamy, D., Grossman, S., \& GLUCK, M. (2004). Impaired probabilistic category learning in hypoxic subjects with hippocampal damage. Neuropsychologia, $\mathbf{4 2}$, 524-535.

Knowlton, B. J., Squire, L. R., \& Gluck, M. A. (1994). Probabilistic classification learning in amnesia. Learning \& Memory, $\mathbf{1}$ 106-120.

Lagnado, D. A., Newell, B. R., Kahan, S., \& Shanks, D. R. (2006). Insight and strategy in multiple-cue learning. Journal of Experimental Psychology: General, 135, 162-183.

Nosofsky, R. M., \& KruschKe, J. K. (2002). Single-system models and interference in category learning: Commentary on Waldron and Ashby (2001). Psychonomic Bulletin \& Review, 9, 169-174.

Poldrack, R. A., Clark, J., Paré-Blagoev, E. J., Shohamy, D., Creso Moyano, J., Myers, C., \& Gluck, M. A. (2001). Interactive memory systems in the human brain. Nature, 414, 546-550.

Sherry, D. F., \& Schacter, D. L. (1987). The evolution of multiple memory systems. Psychological Review, 94, 439-454.

Shohamy, D., Myers, C. E., Onlaor, S., \& Gluck, M. A. (2004). Role of the basal ganglia in category learning: How do patients with Parkinson's disease learn? Behavioral Neuroscience, 118, 676-686.
SQuire, L. R. (2004). Memory systems of the brain: A brief history and current perspective. Neurobiology of Learning \& Memory, 82, 171-177.

WALDRon, E. M., \& Ashby, F. G. (2001). The effects of concurrent task interference on category learning: Evidence for multiple category learning systems. Psychonomic Bulletin \& Review, 8, 168-176.

ZAKI, S. R. (2004). Is categorization performance really intact in amnesia? A meta-analysis. Psychonomic Bulletin \& Review, 11, 1048-1054.

Zeithamova, D., \& Maddox, W. T. (2006). Dual-task interference in perceptual category learning. Memory \& Cognition, 34, 387-398.

\section{NOTES}

1. We thank Rob Nosofsky for suggesting this analysis

2. Analysis of individual card ratings revealed that $43.7 \%$ of participants in the feedback group, compared with only $18.1 \%$ of participants in the observation group, provided ratings (at the end of 102 trials) that were within five units of the objective values for at least one card (e.g., between .15 and .25 for Card 1). In Experiment 1, the respective values were $41.6 \%$ for no load and $16 \%$ for load.

(Manuscript received April 27, 2006; revision accepted for publication August 7, 2006.) 